\section{AI zooms in on highly influential citations}

The number of times a paper is cited is a poor proxy for its impact (see P. Stephan et al. Nature 544, 411-412; 2017). I suggest relying instead on a new metric that uses artificial intelligence (AI) to capture the subset of an author's or a paper's essential and therefore most highly influential citations.

Academics may cite papers for non-essential reasons - out of courtesy, for completeness or to promote their own publications. These superfluous citations can impede literature searches and exaggerate a paper's importance.

The scientific search engine, Semantic Scholar, is the first to automatically identify the subset of a paper's citations in which the paper had a strong impact on the citing work (see http://semanticscholar.org). It further ranks these according to their estimated impact by using machine-learning methods (see go.nature.com/2th2voa). Although still far from perfect, this 'highly influential citations' metric is a substantially better indicator of impact than raw citation counts are. An author's highly influential citation count is simply the sum of the highly influential citations of his or her papers.

This metric and its implementation exemplify the potential of AI to overcome information overload in the research literature.

Oren Etzioni Allen Institute for Artificial Intelligence, Seattle, Washington, USA. orene@allenai.org

\section{Japan disregards whaling review again}

Japan has continued to kill whales since the 1986 commercial whaling moratorium, purportedly for research under Article VIII of the International Convention for the Regulation of Whaling. Last month, the country issued permits for a new scientific whaling programme (NEWREP-NP) in the North Pacific. This is despite the assessment by an International Whaling Commission (IWC) expert panel in February 2017 that Japan's lethal sampling remains unjustified.

The panel also concluded that the NEWREP-NP proposal gave insufficient justification for sampling design and sample size, and failed to show that additional age data from dead whales could improve management of whale stocks. There were further concerns about the impact of proposed catches on minke whales around Japan and South Korea - populations that are already threatened by high fisheries bycatch. The panel therefore advised that NEWREP-NP should not start, pending further study. In May, the IWC Scientific Committee endorsed the panel's recommendations.

A previous expert panel reached similar conclusions in 2015 about Japan's Antarctic scientific whaling programme, NEWREP-A (see A.S. Brierley and P. J. Clapham Nature 529, 283; 2016, and J. Morishita Nature 531, 35; 2016). As with NEWREP-NP, this went ahead anyway, despite the availability of widely used non-lethal alternatives for acquiring the information needed for stock management.

Phillip J. Clapham* Alaska Fisheries Science Center, Seattle, Washington, USA.

phillip.clapham@noaa.gov

${ }^{*}$ Supported by 40 signatories

(listed at go.nature.com/2tsjzew).

\section{Flagging gender bias doesn't always work}

As a female scientist, I find it demotivating to read wellintended articles that reiterate examples of gender bias and the extra pressures on women scientists. In my view, a change in perspective is called for.

Gender bias and sexism occur in many different disciplines and settings. Statistics and behavioural studies confirm that women are under-represented in science because many leave their jobs before they reach senior academic positions.

The fact that women have a statistically lower chance of success in research exacerbates the huge stress that besets any scientist as a result of the pressures to publish, to acquire funding, to teach and to specialize. Journals and magazines rarely miss a chance to remind women how hostile some environments can be to them, even when they are well-equipped to pursue a career in science (see, for example, Nature 541, 455-457; 2017). I personally find such reminders intimidating.

I suggest instead that focused networking events for women and publicizing the achievements of working female scientists would help to overcome the isolation of women academics and make them feel more a part of the scientific community.

Greta Faccio St Gallen, Switzerland. greta.faccio@gmail.com

\section{Stop ruining Turkey's geological heritage}

The United Nations Educational, Scientific and Cultural Organization (UNESCO) designated the Quaternary Kula region in western Turkey as a geopark - a geological heritage of outstanding international significance - in 2013. The aim was to protect this rare and beautiful site of extinct volcanoes and to make it accessible to the public. A UNESCO inspection this month will find that the geopark is instead being degraded at an alarming rate.

Rubbish from Kula town has been dumped in the pristine volcanic cones, shanty buildings have been allowed to encroach on the lava fields and construction refuse has piled up near the geopark walkways. The crater of the only perfectly preserved cone, Sandal, is polluted by debris from tourists.

Pumice quarrying in the 1980s had already destroyed one-quarter of the magnificent Kula cone, which is now filled with municipal waste. Quarrying continues to wreck other cones.

We urge UNESCO to call the Turkish culture ministry to task and stop the ignorant, wanton destruction of this precious natural and cultural legacy. A. M. Celal Şengör, Nalan Lom Istanbul Technical University, Turkey.

sengor@itu.edu.tr

\section{Defend real science in the Balkans}

Antiscience and pseudoscience seem to be rife in some nations of the former Yugoslavia, and have potentially harmful consequences. As one of the few science journalists in Bosnia and Herzegovina, I urge the region's researchers to speak out and boost the international scientific standing of their countries.

A worrying instance of antiscience is the widely publicized petition organized in Serbia in May (see Nature 545, 138-139; 2017). Signed by engineers, physicians and other academics, it calls for schools and universities to teach 'alternative theories' of evolution such as intelligent design and creationism. The organizers back their cause with claims that evolution is now being discredited by "more and more famous scientists" worldwide.

In my view, this petition is a scientific humiliation for Serbia. Croatia and Bosnia and Herzegovina also have advocates of intelligent design. Those who disagree will not speak up, fearing retribution and public outcry. Lift this shameful silence so that the region's science can flourish again. Jelena Kalinić Sarajevo, Bosnia and Herzegovina. jkalinic10@gmail.com 